$$
\left(\begin{array}{ll}
N & 0 \\
0 & 0
\end{array}\right)
$$

with $N$ of type $(r, r)$ has been reached. This, according to (a), completes the proof.

College of the City of New York

\title{
THE GEOMETRY OF VELOCITY SYSTEMS
}

EDWARD KASNER AND JOHN DECICCO

1. Introduction. In connection with the investigation of the differential geometric properties of positional fields of force, Kasner introduced certain important systems of curves which he termed velocity systems. ${ }^{1}$ In this paper, we propose to present some of the old and many new geometric properties of any velocity system.

Since velocity systems serve to characterize the conformal group, many of our results are logically theorems of conformal geometry. This may be contrasted with the study of all the trajectories of positional fields of force, which is essentially differential projective geometry.

2. Velocity systems. We shall find it most convenient for our study to use the minimal coordinates $u=x+i y, v=x-i y, p=d v / d u$, $q=d^{2} v / d u^{2}$, where, of course, $(x, y)$ are the cartesian coordinates of the plane.

In the plane, consider a particle of unit mass moving in any field of force whose components parallel to the $u$-axis and the $v$-axis are $d(u, v)$ and $c(u, v)$. The equations of motion are

$$
\frac{d^{2} u}{d t^{2}}=d(u, v), \quad \frac{d^{2} v}{d t^{2}}=c(u, v),
$$

where $t$ is the time. Now if $r$ is the radius of curvature along a trajectory, we have

$$
\omega^{2}=r N \text {, }
$$

Presented to the Society, February 22,1941, under the title Conformal geometry of velocity systems; received by the editors June 23, 1942.

${ }^{1}$ Kasner, Differential-geometric aspects of dynamics, Amer. Math. Soc. Colloquium Publications vol. 3 (1912); Trans. Amer. Math. Soc. vols. 9-10 (1908-1909). 
where $\omega=p^{1 / 2} d u / d t$ is the velocity (speed) and $N$ is the principal normal component of the force given by

$$
N=\frac{c-d p}{2 i p^{1 / 2}} .
$$

Since (1) defines a three-parameter family of curves, the path is uniquely determined if the initial position, the initial direction, and the initial velocity are given.

If only the initial position and the initial direction of motion are given, then the initial radius of curvature will depend on the initial velocity. In the actual trajectory, the velocity $\omega$ varies from point to point. If now the square of the velocity $\omega^{2}$ is replaced by some constant, say $1 /$ const., the resulting equation is

$$
q=\text { const. } p(c-d p) \text {. }
$$

The curves satisfying this differential equation, they are not in general trajectories, are called velocity curves.

For any field, a curve is a velocity curve corresponding to the speed $\omega_{0}$, provided any particle starting from a lineal element of the curve with that speed, describes a trajectory which is initially osculated by that curve. In a given field of force, there are in all $\infty^{3}$ trajectories and $\infty^{3}$ velocity curves.

If the constant is given, there are $\infty^{2}$ velocity curves. In particular, if the constant and hence the velocity is taken to be unity, the following result is obtained.

Theorem 1. Any system of $\infty^{2}$ curves defined by a differential equation of the form ${ }^{2}$

$$
q=p(c-d p)
$$

may be regarded as the totality of velocity curves corresponding to unit velocity in the unique field of force whose u-component is $d(u, v)$ and whose v-component is $c(u, v)$.

Throughout this paper, we shall understand a velocity system to be the totality of velocity curves with unit velocity. That is, any velocity system is given by the preceding differential equation.

Theorem 2. Property A. A set of $\infty^{2}$ curves of the plane is a velocity system if and only if the $\infty^{1}$ osculating circles of the $\infty^{1}$ curves of the set passing through any point $(u, v)$, constructed at $(u, v)$, form a linear pencil of circles.

${ }^{2}$ The corresponding cartesian equation of the general velocity system is $y^{\prime \prime}=\left(1+y^{\prime 2}\right)\left(\chi-y^{\prime} \phi\right)$ where $\phi$ and $\chi$ are any functions of $(x, y)$. 
Of course, the centers of these circles describe a straight line. The other point of intersection $(U, V)$ of this pencil of circles is

$$
U=u+2 / c, \quad V=v+2 / d .
$$

We shall call this the associated correspondence of the velocity system.

Obviously any arbitrary point transformation except identity, may serve as the associated correspondence of a unique velocity system.

Special important types of velocity systems are natural families, isogonal families, and $\Gamma$ families of curves. Now we shall consider these sets of curves (special wexes).

3. Natural families. A natural family consists of the extremals connected with the variation problem of the form

$$
\int F(u, v) d s=\text { minimum }
$$

where $F$ is a point function and $d s=p^{1 / 2} d u$ is the element of arc. Any natural family is a velocity system for which $c_{v}=d_{u}$.

Kasner has already indicated the many problems which are connected with natural families. Thus natural families arise in the study of (a) the trajectories of a conservative field of force, (b) general catenaries, (c) the paths of light in an isotropic medium, and (d) the geodesics of a surface. ${ }^{1}$ (See papers by Lipka, Douglas, Blaschke, Fialkow, DeCicco, Struik.)

A natural family of curves is a special type of velocity system. Hence in addition to the Property A, it must possess an additional differential property.

Theorem 3. Property B. At any point $(u, v)$, there exist two lineal elements $E_{1}$ and $E_{2}$, each of which is converted by the associated correspondence of the velocity system into a cocircular element at the point $(U, V)$. Our velocity system is a natural family if and only if $E_{1}$ and $E_{2}$ are orthogonal.

Thus any system of $\infty^{2}$ curves with the properties $A$ and $B$ is a natural family.

4. Isogonal families. Any nonminimal simple family of $\infty^{1}$ curves is given by a differential equation of the first order $p=e^{f(u, v)}$. The set of all simple families of curves each of which makes a constant angle with all the curves of the given simple family is called a complete isogonal family. Any isogonal family is a velocity system for which $c=f_{u}$ and $d=-f_{v}$ so that $c_{v}=-d_{u}$. 
Theorem 4. Property C. At any point $(u, v)$, there exist two lineal elements $E_{1}$ and $E_{2}$, each of which is converted by the associated correspondence of the velocity system into an element orthogonal to the cocircular element at the point $(U, V)$. Our velocity system is an isogonal family if and only if $E_{1}$ and $E_{2}$ are othogonal.

Thus the conditions necessary and sufficient for an isogonal family are properties $\mathrm{A}$ and $\mathrm{C}$. Thus the famous theorem of Cesàro-Scheffers is completed.

5. $\Gamma_{0}$ systems. The search for those velocity systems which are both natural and isogonal yields the following result.

THEOREM 5. For a velocity system to be both natural and isogonal (that is, to possess properties $\mathrm{A}, \mathrm{B}$, and $\mathrm{C}$ ), it is necessary and sufficient that it be the complete set of isogonal trajectories of an isothermal family.

This family may be called a conformal rectilinear wex. ${ }^{3}$ That is, it is conformally equivalent to the $\infty^{2}$ straight lines of the plane. Any such system shall be denoted by $\Gamma_{0} \cdot{ }^{4}$ Any system $\Gamma_{0}$ may be characterized geometrically as a velocity system whose associated point correspondence is direct conformal.

6. The $\Gamma$ families. Any set of $\infty^{2}$ curves which is conformally equivalent to the set of $\infty^{2}$ circles orthogonal to a fixed proper circle (or a fixed straight line) is called a $\Gamma$ family. ${ }^{5}$

The finite form of a $\Gamma$ family is

$$
a_{0} \phi(u) \psi(v)+b_{0}[\phi(u)+\psi(v)]+c_{0}=0 .
$$

Upon eliminating the constants, $a_{0}, b_{0}, c_{0}$, we discover that a $\Gamma$ family is a velocity system where the basic functions

$$
c=\frac{\phi_{u u}}{\phi_{u}}-\frac{2 \phi_{u}}{\phi-\psi}, \quad d=\frac{\psi_{v v}}{\psi_{v}}+\frac{2 \psi_{v}}{\phi-\psi} .
$$

Now we proceed to find the conditions on the functions $c$ and $d$ that a velocity system be a $\Gamma$ family. Solving the first equation for $\psi$,

${ }^{3}$ Kasner has introduced the word wex to represent any system of $\infty^{2}$ curves. Thus a wex is given by a differential equation of the second order $y^{\prime \prime}=f\left(x, y, y^{\prime}\right)$. A web denotes $n \infty^{1}$ curves.

${ }^{4}$ The authors have determined all curvature element transformations which preserve the class of $\Gamma_{0}$ systems. See Kasner and DeCicco, Transformation theory of the isogonal trajectories of an isothermal family, Proc. Nat. Acad. Sci. U.S.A., vol. 28 (1942).

${ }^{5}$ The $\Gamma$ and $\Gamma_{0}$ systems are special subsets of $\Omega$ families. Any $\Omega$ family is conformally equivalent to the $\infty^{3}$ circles. See Kasner and DeCicco, Families of curves conformally equivalent to circles, Trans. Amer. Math. Soc. vol. 49 (1941) pp. 378-391. 
and the second for $\phi$, we find

$$
\psi=\phi+\frac{2 \phi_{u}}{c-\phi_{u u} / \phi_{u}}, \quad \phi=\psi+\frac{2 \psi_{v}}{d-\psi_{v v} / \psi_{v}} .
$$

By differentiating the first equation of (10) with respect to $u$ and the second with respect to $\nu$, we obtain

(11) $\quad 2 c_{u}-c^{2}=\frac{2 \phi_{\mu} \phi_{u u u}-3 \phi_{u u}^{2}}{\phi_{u}^{2}}, \quad 2 d_{v}-d^{2}=\frac{2 \psi_{v} \psi_{v v v}-3 \psi_{v v}^{2}}{\psi_{v}^{2}}$.

Substituting the values of $\phi$ and $\psi$ as determined by (10) into these equations, we find

$$
d c_{v}=c_{v v}, \quad c d_{u}=d_{u u} .
$$

Theorem 6. Property D. $A$ velocity system represents a $\Gamma$ family of curves if and only if the functions $c$ and $d$ with $c_{v} \neq 0$ and $d_{u} \neq 0$ satisfy either one of the three equivalent systems of partial differential equations of the second order.

$$
\begin{gathered}
c_{u v}=c c_{v}, \quad c_{v v}=d c_{v} ; \\
d_{u v}=d d_{u}, \quad d_{u u}=c d_{u} ; \\
c_{v}=d_{u}, \quad c_{u v}=c c_{v}, \quad d_{u v}=d d_{u} .
\end{gathered}
$$

The last set of equations demonstrates that every $\Gamma$ family is a natural family. But the converse is not true. The only possible $\Gamma$ families which are isogonal are the $\Gamma_{0}$ systems.

7. Velocity systems which possess isothermal families. We shall now classify velocity systems according to the number of isothermal families they contain. For the velocity system (5) to contain the simple family $p=e^{f(u, v)}$, it is necessary and sufficient that

$$
f_{u}+e^{f} f_{v}=c-e^{f} d .
$$

If the simple family is isothermal, then, as deduced from the theorem of Lie, $f_{u v}=0$. Differentiating the above partially with respect to $v$ and simplifying, we find

$$
f_{v v}+f_{v}^{2}+d f_{v}=e^{-f} c_{v}-d_{v}
$$

Differentiating this partially with respect to $u$ and making use of (14), we obtain

$$
\left(c_{v}-d_{u}\right) f_{v}=\left(d_{u v}-d c_{v}\right)-\left(c_{u v}-c c_{v}\right) e^{-\uparrow} .
$$


From these equations, it is concluded that a velocity system will contain exactly $\infty^{2}$ isothermal systems if and only if it is a $\Gamma$ family. This is a characteristic property of $\Gamma$ families (including $\Gamma_{0}$ ).

Suppose next our velocity system is natural but not a $\Gamma$ family. Then there exists possibly one isothermal family of curves. This possible isothermal family is

$$
p=\frac{c_{u v}-c c_{v}}{d_{u v}-d c_{v}} \text { where } c_{v}=d_{u}
$$

Finally let us suppose that our family is not natural. Such a velocity system may possess at most $\infty^{1}$ isothermal families. These are defined by (14) and (16).

Differentiating the equation (16) with respect to $u$ and eliminating $f_{u}$ and $f_{v}$ from the result, we find

$$
e^{f}=\frac{\left(\frac{\partial}{\partial u} \frac{c_{u v}-c c_{v}}{c_{v}-d_{u}}\right)-\left(\frac{c_{u v}-c c_{v}}{c_{v}-d_{u}}\right)^{2}-\frac{c\left(c_{u v}-c c_{v}\right)}{c_{v}-d_{u}}}{\left(\frac{\partial}{\partial u} \frac{d_{u v}-d c_{v}}{c_{v}-d_{u}}\right)-\frac{\left(c_{u v}-c c_{v}\right)\left(d_{u v}-d c_{v}\right)}{\left(c_{v}-d_{u}\right)^{2}}-\frac{d\left(c_{u v}-c c_{v}\right)}{c_{v}-d_{u}}} .
$$

Again by differentiating (16) with respect to $v$ and eliminating $f_{u}, f_{v}, f_{v v}$ from the result, we find

$$
e^{f}=-\frac{\left(\frac{\partial}{\partial v} \frac{c_{u v}-c c_{v}}{c_{v}-d_{u}}\right)+\frac{\left(c_{u v}-c c_{v}\right)\left(d_{u v}-d c_{v}\right)}{\left(c_{v}-d_{u}\right)^{2}}+\frac{d\left(c_{u v}-c c_{v}\right)}{c_{v}-d_{u}}+c_{v}}{\left(\frac{\partial}{\partial v} \frac{d_{u v}-d c_{v}}{c_{v}-d_{u}}\right)+\left(\frac{d_{u v}-d c_{v}}{c_{v}-d_{u}}\right)^{2}+\frac{d\left(d_{u v}-d c_{v}\right)}{c_{v}-d_{u}}+d_{v}} .
$$

THEOREM 7. A velocity system of curves may contain exactly $\infty^{2}, \infty^{1}$, one, or no isothermal systems as described below.

(1) A velocity system will contain exaclly $\infty^{2}$ isothermal families if and only if it is a $\Gamma$ family (including $\Gamma_{0}$ ).

(2) A nonnatural velocity system will contain exactly $\infty^{1}$ isothermal systems if and only if the functions $c$ and $d$ satisfy the four partial differential equations of the third order obtained by setting equal to zero the numerators and denominators of the fractions in (18) and (19).

(3) A nonnaturäl velocity system will contain exactly one isothermal system if the functions $c$ and $d$ satisfy one partial differential equation of the third order (obtained by the equalities of the right- 
hand sides of (18) and (19), one of the fourth order (the family (18) or (19) lies in the velocity system), and one of the fifth order (the family (18) or (19) is isothermal).

A natural family will contain exactly one isothermal family if the functions $c$ and $d$ satisfy identically one partial differential equation of third order (the family (17) must lie in the velocity system), and another of fourth order (the family (17) must be isothermal).

(4) All other types of velocity systems contain no isothermal families. This is of course the general case.

Theorem 8. Property E. $A$ velocity system is a $\Gamma$ or $\Gamma_{0}$ family if and only if it contains $\infty^{2}$ isothermal families.

Properties A and E are necessary and sufficient conditions for the characterization of a $\Gamma$ or $\Gamma_{0}$ family.

8. Reciprocal velocity systems. Let $\Sigma$ be the associated point correspondence (6) of a velocity system $S$. The unique velocity system $S^{*}$ whose associated point correspondence is the inverse $\Sigma^{-1}$ of $\Sigma$ is said to be the reciprocal 6 or conjugate of $S$.

By (7), we find that the reciprocal velocity system $S^{*}$ of $S$ is

$$
Q=P(\gamma-\delta P),
$$

where

$$
\begin{aligned}
\gamma & =-c, \quad \delta=-d, \\
\frac{\partial \gamma}{\partial U} & =-\frac{c^{2}}{J}\left[c_{u}\left(d^{2}-2 d_{v}\right)+2 c_{v} d_{u}\right], \quad \frac{\partial \gamma}{\partial V}=-\frac{c^{2} d^{2} c_{v}}{J}, \\
\frac{\partial \delta}{\partial U} & =-\frac{c^{2} d^{2} d u}{J}, \quad \frac{\partial \delta}{\partial V}=-\frac{d^{2}}{J}\left[2 d_{u} c_{v}+d_{v}\left(c^{2}-2 c_{u}\right)\right], \\
J & =\left(c^{2}-2 c_{u}\right)\left(d^{2}-2 d_{v}\right)-4 d_{u} c_{v} .
\end{aligned}
$$

THEOREM 9. The reciprocal velocity system $S^{*}$ of a given velocity system $S$ is natural or isogonal according as $S$ is natural or isogonal.

The reciprocity is not valid in general for $\Gamma$ systems (of course excluding $\Gamma_{0}$ ). We now wish to find those particular velocity systems whose reciprocals are $\Gamma$ families.

Before proceeding, let us note the following formulas in addition to those given by (21)

\footnotetext{
${ }^{6}$ Scheffers, Math. Ann. vol. 60 (1905) pp. 491-531.
} 


$$
\begin{aligned}
\frac{\partial^{2} \gamma}{\partial U \partial V} / \frac{\partial \gamma}{\partial V}=\frac{2 c}{J} & {\left[c_{u}\left(d^{2}-2 d_{v}\right)+2 c_{v} d_{u}\right]+\frac{2 c^{2} d d_{u}}{J} } \\
& +\frac{c^{2}}{J c_{v}}\left[c_{u v}\left(d^{2}-2 d_{v}\right)+2 d_{u} c_{v v}\right] \\
& -\frac{c^{2}}{J^{2}}\left[J_{u}\left(d^{2}-2 d_{v}\right)+2 J_{v} d_{u}\right],
\end{aligned}
$$

$$
\begin{aligned}
\frac{\partial^{2} \delta}{\partial U \partial V} / \frac{\partial \delta}{\partial U}= & \frac{2 c d^{2} c_{v}}{J}+\frac{2 d}{J}\left[2 c_{v} d_{u}+d_{v}\left(c^{2}-2 c_{u}\right)\right] \\
& +\frac{d^{2}}{J d_{u}}\left[2 c_{v} d_{u u}+d_{u v}\left(c^{2}-2 c_{u}\right)\right] \\
& -\frac{d^{2}}{J^{2}}\left[2 J_{u} c_{v}+J_{v}\left(c^{2}-2 c_{u}\right)\right] .
\end{aligned}
$$

By (21) and (22), we may prove the following result.

THEOREM 10. A velocity system $S$ is the reciprocal of a $\Gamma$ family $S^{*}$ if and only if its functions $c$ and $d$ with $c_{v} \neq 0$ and $d_{u} \neq 0$ satisfy the partial differential equations of third order

$$
c_{v}=d_{u}, \quad \frac{J_{u}}{J}=c+\frac{c_{u v}}{c_{v}}, \quad \frac{J_{v}}{J}=d+\frac{c_{v v}}{c_{v}} .
$$

In the real domain, it may be proved that the only velocity systems such that both $S$ and $S^{*}$ are $\Gamma$ families must consist of the $\infty^{2}$ circles orthogonal to a fixed circle. In that event, $S$ and $S^{*}$ coincide.

9. The conformal covariants of a velocity system. ${ }^{7}$ Upon extending a conformal transformation twice, we find the formulas

$$
U=\phi(u), V=\psi(v), P=\frac{\psi_{v}}{\phi_{u}} p, \frac{Q}{P}=\frac{q}{\phi_{u} p}+\frac{\psi_{v v} p}{\phi_{u} \psi_{v}}-\frac{\phi_{u u}}{\phi_{u}^{2}},
$$

which have many applications.

THEOREM 11. Every velocity system $S(c, d)$ is converted into a velocity system $\Sigma(C, D)$ by a conformal transformation. The transformation formulas between corresponding velocity systems $S$ and $\Sigma$ are

7 For the analogous conformal geometry of a simple family of curves, see DeCicco, The two conformal covariants of a field, Revista di Matematica vol. 2 (1941) pp. 59-66. A paper on Isothermal families will appear in the forthcoming Pastor commemoration volume edited by Beppo Levi. 


$$
C=\frac{c}{\phi_{u}}-\frac{\phi_{u u}}{\phi_{u}^{2}}, \quad D=\frac{d}{\psi_{v}}-\frac{\psi_{v v}}{\psi_{v}^{2}} .
$$

(It may be proved that if a quasi-contact transformation of curvature elements carries every velocity system into a velocity system, then it must be a conformal transformation.)

We now inquire: what are the necessary and sufficient conditions that the velocity systems $S(c, d)$ and $\Sigma(C, D)$ be equivalent by a conformal transformation? That is, given the four functions $c(u, v)$, $d(u, v), C(\phi, \psi), D(\phi, \psi)$, can we find $\phi(u)$ and $\psi(v)$ such that the equations (25) are satisfied?

Firstly upon differentiating the first equation (25) with respect to $v$ and the second with respect to $u$, we find

$$
\phi_{u} \psi_{v}=\frac{c_{v}}{C_{\psi}}=\frac{d_{u}}{D_{\phi}} .
$$

Differentiating this with respect to $u$ and $v$ and eliminating $\phi_{u u}$ and $\psi_{v v}$ by (25), we obtain

$$
\phi_{u}=\left(\frac{c_{u v}}{c_{v}}-c\right) /\left(\frac{C_{\phi \psi}}{C_{\psi}}-C\right), \psi_{v}=\left(\frac{d_{u v}}{d_{u}}-d\right) /\left(\frac{D_{\phi \psi}}{D_{\phi}}-D\right) .
$$

Now these equations will determine appropriate functions $\phi_{u}$ and $\psi_{v}$ if and only if the derivative of the first and second fractions with respect to $v$ and $u$, respectively, are zero. Accordingly we find that the velocity system $S(c, d)$ possesses the two absolute covariants

$$
\begin{aligned}
& {\left[\frac{\partial}{\partial v} \log \left(\frac{c_{u v}}{c_{v}}-c\right)\right] /\left(\frac{d_{u v}}{d_{u}}-d\right) \quad \text { and }} \\
& {\left[\frac{\partial}{\partial u} \log \left(\frac{d_{u v}}{d_{u}}-d\right)\right] /\left(\frac{c_{u v}}{c_{v}}-c\right) .}
\end{aligned}
$$

But these values as given by (27) must also satisfy the equations (25) and (26). Hence our velocity system $S(c, d)$ possesses the three absolute covariants

$$
\begin{gathered}
\left(\frac{c_{u v}}{c_{v}}-c\right)\left(\frac{d_{u v}}{d_{u}}-d\right) / c_{v} \\
{\left[c-\frac{\partial}{\partial u} \log \left(\frac{c_{u v}}{c_{v}}-c\right)\right] /\left(\frac{c_{u v}}{c_{v}}-c\right) \text { and }} \\
{\left[d-\frac{\partial}{\partial v} \log \left(\frac{d_{u v}}{d_{u}}-d\right)\right] /\left(\frac{d_{u v}}{d_{u}}-d\right) .}
\end{gathered}
$$


Summarizing, we have this theorem.

THEOREM 12. The velocity system $S(c, d)$ possesses the six absolute covariants

$$
\begin{gathered}
\frac{c_{v}}{d_{u}}, \quad\left(\frac{c_{u v}}{c_{v}}-c\right)\left(\frac{d_{u v}}{d_{u}}-d\right) / c_{v}, \\
{\left[\frac{\partial}{\partial v} \log \left(\frac{c_{u v}}{c_{v}}-c\right)\right] /\left(\frac{d_{u v}}{d_{u}}-d\right),} \\
{\left[\frac{\partial}{\partial u} \log \left(\frac{d_{u v}}{d_{u}}-d\right)\right] /\left(\frac{c_{u v}}{c_{v}}-c\right),} \\
{\left[c-\frac{\partial}{\partial u} \log \left(\frac{c_{u v}}{c_{v}}-c\right)\right] /\left(\frac{c_{u v}}{c_{v}}-c\right),} \\
{\left[d-\frac{\partial}{\partial v} \log \left(\frac{d_{u v}}{d_{u}}-d\right)\right] /\left(\frac{d_{u v}}{d_{u}}-d\right) .}
\end{gathered}
$$

Any conformal covariant of a velocity system is a function of these and their conformal partial derivatives. These are defined by

$$
\frac{\partial}{\partial u} /\left(\frac{c_{u v}}{c_{v}}-c\right) \quad \text { and } \quad \frac{\partial}{\partial v} /\left(\frac{d_{u v}}{d_{u}}-d\right) .
$$

Let now $S(c, d)$ and $\Sigma(C, D)$ be such that there exist functions $\phi$ and $\psi$ which satisfy the resulting covariant equations (30). If the operations on these six equations with the conformal derivatives (31) are reduced to identities by these functions $\phi$ and $\psi$, then $S(c, d)$ and $\Sigma(C, D)$ are conformally equivalent.

Columbia University and

ILLINOIS INSTITUTE OF TECHNOLOGY 